\title{
Biotechnical paving of recombinant enterocin A as the candidate of anti-Listeria agent
}

\author{
Xiaoyuan $\mathrm{Hu}^{1,2,3 \dagger}$, Ruoyu Mao ${ }^{1,2+}$, Yong Zhang ${ }^{1,2+}$, Da Teng ${ }^{1,2}$, Xiumin Wang ${ }^{1,2}$, Di Xi ${ }^{1,2}$, Jianzhong Huang ${ }^{3^{*}}$
} and Jianhua Wang ${ }^{1,2^{*}}$

\begin{abstract}
Background: Enterocin A is a classic lla bacteriocin isolated firstly from Enterococcus faecium CTC492 with selective antimicrobial activity against Listeria strains. However, the application of enterocin A as an anti-Listeria agent has been limited due to its very low native yield. The present work describes high production of enterocin A through codon optimization strategy and its character study.

Results: The gene sequence of enterocin A was optimized based on preferential codon usage in Pichia pastoris to increase its expression efficiency. The highest anti-Listeria activity reached 51,200 AU/ml from $180 \mathrm{mg} / \mathrm{l}$ of total protein after $24 \mathrm{~h}$ of induction in a 5 - $\mathrm{L}$ fermenter. Recombinant enterocin A ( $\mathrm{rEntA}$ ), purified by gel filtration chromatography, showed very strong activity against Listeria ivanovii ATCC 19119 with a low MIC of $20 \mathrm{ng} / \mathrm{ml}$. In addition, the rEntA killed over $99 \%$ of tested L. ivanovii ATCC19119 within $4 \mathrm{~h}$ when exposed to $4 \times \mathrm{MIC}(80 \mathrm{ng} / \mathrm{ml})$. Moreover, it showed high stability under a wide pH range (2-10) and maintained full activity after $1 \mathrm{~h}$ of treatment at $80^{\circ} \mathrm{C}$ within a pH range of 2-8. Its antimicrobial activity was enhanced at 25 and $50 \mathrm{mM} \mathrm{NaCl}$, while 100-400 mM $\mathrm{NaCl}$ had little effect on the bactericidal ability of rEntA.
\end{abstract}

Conclusion: The EntA was successfully expressed in P. pastoris, and this feasible system could pave the pre-industrial technological path of rEntA as a competent candidate as an anti-Listeria agent. Furthermore, it showed high stability under wide ranges of conditions, which could be potential as the new candidate of anti-Listeria agent.

Keywords: Enterocin A, Listeria ivanovii, Antimicrobial activity, Pichia pastoris, Expression

\section{Background}

Bacteriocins are antimicrobial peptides synthesized in the ribosome and secreted into medium to establish a competitive advantage in their environment by eliminating competitors to gain resources [1]. Bacteriocins are generally classified in terms of size, structure, and modifications. Class I bacteriocins are lantibiotics. Class II bacteriocins consist of small peptides that do not contain modified residues. Class III bacteriocins usually are large and heat-labile proteins [2]. The well-known bacteriocin is nisin, a class I bacteriocin, which is widely used in commerce [3]. Recently, many reports clearly

\footnotetext{
*Correspondence: hjz@fjnu.edu.cn; 2681298635@qq.com

${ }^{\dagger}$ Equal contributors

${ }^{3}$ College of Life Science, Engineering Research Center of Industrial Microbiology, Fujian Normal University, Fuzhou 350108, China

'Key Laboratory of Feed Biotechnology, Ministry of Agriculture, Beijing

100081, China

Full list of author information is available at the end of the article
}

indicate that bacteriocins of class IIa have greater potential as antimicrobial agents [4] with a narrower inhibitory spectrum to Listeria strains than nisin [5]. Listeria, the most common pathogen in food, can lead the host to suffer from serious diseases such as enteritis, sepsis, meningitis and abortion [6]. The mortality rate caused by listeriosis is between 15 and 30\% [7,8]. Additionally, some strains of $L$. monocytogenes easily acquire resistance to many antibiotics [9]. To control food contamination and listeriosis effectively, more or better anti-listerial drugs are needed.

Enterocin A (EntA), with many antimicrobial merits, is a class IIa bacteriocin that was first isolated from Enterococcus faecium CTC492 in the mid-1990s. Its mature form is composed of 47 amino acids with two disulfide bridges [10]. It shows high activity, particularly against Listeria species at nanomolar concentrations [11]. The native EntA has proven to effectively inhibit $L$. monocytogenes in fermented foods $[12,13]$. However, the low 
levels of bacteriocins secreted from natural strains do not meet the requirements of the industrial scale and have limited its application to study stages thus far. Therefore, various heterologous expressions were attempted in lactic acid bacteria, Escherichia. coli (E.coli) and yeast [12,14-16], but their actual production levels were not desirable and left room for improvement. Pichia pastoris is considered to be a promising system because the target protein can be directly secreted into culture medium. It was reported that the production and bactericidal titer of enterocin $\mathrm{P}$ expressed by $P$. pastoris $\mathrm{X}-33$ was 3.7 - and 16-fold higher $(28.2 \mu \mathrm{g} / \mathrm{ml}$ and 1,024 $\mathrm{BU} / \mathrm{ml})$, respectively, than that from the native E. faecium P13 [17]; in fact, even though the level of $45.1 \mu \mathrm{g} / \mathrm{ml}$ of recombinant enterocin A expressed by $P$. pastoris [18] was still too low for its industrial production and end application, it demonstrates the potential to increase its productivity to be as high as possible and to further easily characterize its purification and properties. However, there are only few studies at the modification of bacteriocin genes, such as gene synthesis or codon optimization, which is considered as a promising technique for increasing protein expression level [19]; thus, further work with this system is necessary to achieve an increased protein expression level of target gene.

Due to the high anti-Lister activity of EntA and its low yield either in native strain and recombinant expression system, the EntA gene was optimized by the preferential codon usage of $P$. pastoris and was expressed into medium as recombinant EntA (rEntA). The purification of rEntA from ferment supernatant was tried by four methods including gel filtration chromatography, then the antimicrobial activity, proteolytic sensibility and stabilities of heat, $\mathrm{pH}$ and salt of purified rEntA were examined.

\section{Results}

Construction and transformation of the expression vector Compared to naturally occurring EntA, the base codons coding for 37 residues $(78.72 \%)$ in total 47 amino acids were optimized by the preferential codon usage of $P$. pastoris (Figure 1A). The GC content of the full target sequence increased from $41.13 \%$ to $41.9 \%$. The gene sequence of the optimized EntA was synthesized and inserted into pPICZ $\alpha \mathrm{A}$ between $\mathrm{XhoI}$ and $\mathrm{XbaI}$ sites (Figure $1 \mathrm{~B}, \mathrm{C}$ ). The expression vector pPICZ $\alpha \mathrm{A}-\mathrm{EntA}$

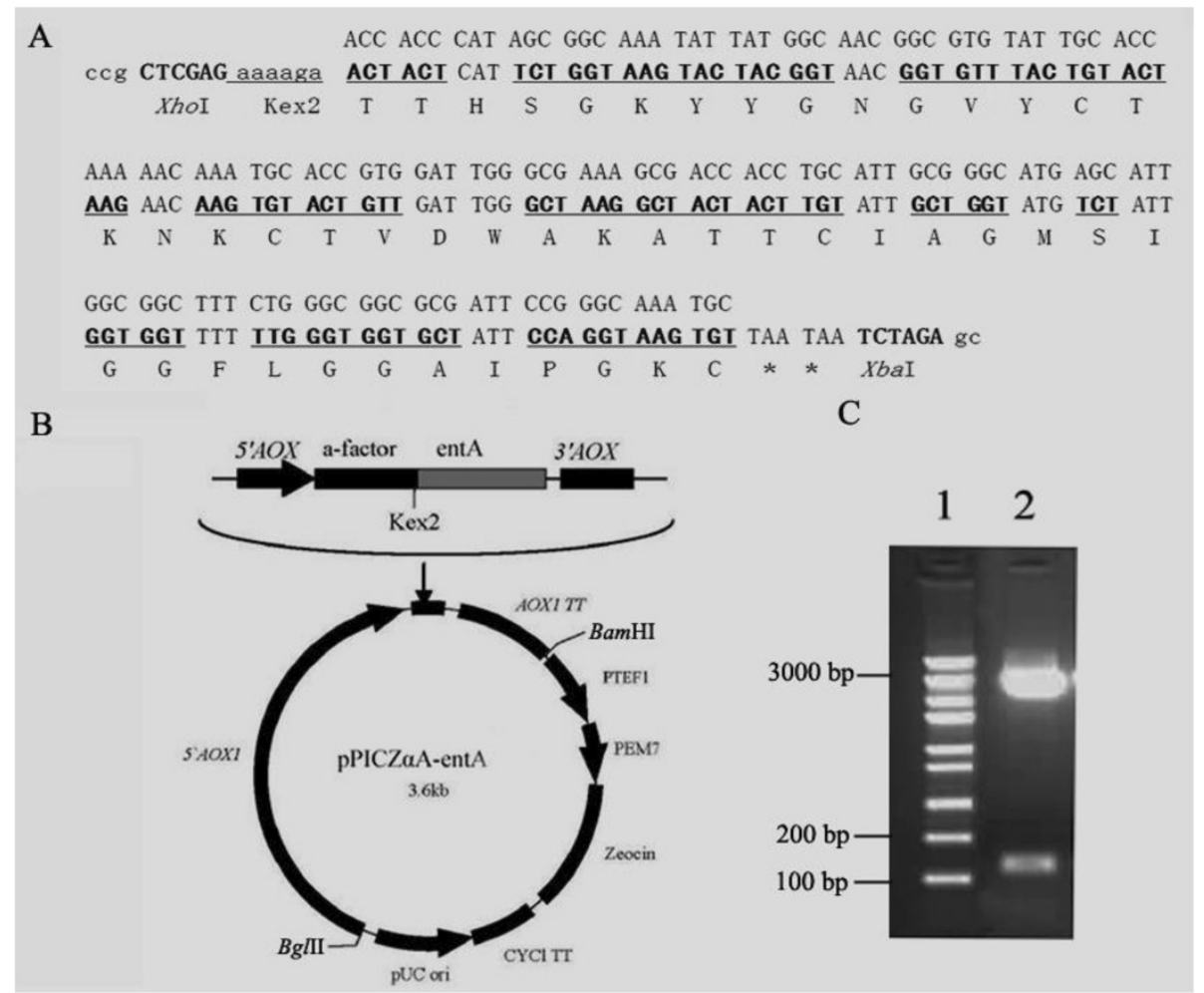

Figure 1 Construction of the expression plasmid pPICZaA-EntA. A, The nucleotide sequence of EntA and its corresponding amino acid sequence. The upper line indicates the wild-type EntA gene sequence. The middle line is the codon-optimized EntA gene sequence. Optimized codons are underlined with boldface type. The lower line represents the amino acid sequence of EntA. The termination codon is marked by an asterisk. B, Map of the recombinant plasmid pPICZaA-EntA. C, Electrophoretic analysis of the recombinant vector containing the EntA gene. Lane 1, DNA marker; lane 2, pPICZaA-EntA digested by Xhol and Xbal. 
was transferred into competent $E$. coli DH5 $\alpha$ cells. Resulting transformants were confirmed by PCR and DNA sequencing. Correct plasmid and control vector pPICZ $\alpha \mathrm{A}$ were linearized by PmeI and transferred into competent $P$. pastoris $\mathrm{X}-33$ cells by electroporation. Positive transformations were screened and confirmed by colony PCR.

\section{Expression of rEntA in shake flasks and at the fermenter level}

The heterologous expression of rEntA in $P$. pastoris $\mathrm{X}$ 33 was induced by methanol at the concentration of $0.5 \%$ and analyzed by agar diffusion and Tricine-SDSPAGE. P. pastoris X-33 containing the empty pPICZ $\alpha \mathrm{A}$ vector was used as a negative control. As shown in Figure $2 \mathrm{~A}$, after $12 \mathrm{~h}$ of methanol induction, the antibacterial activity of the supernatants of $P$. pastoris $\mathrm{X}-33$ (pPICZ $\alpha \mathrm{A}-$ EntA) was observed. Its antibacterial activity reached maximum with $6,400 \mathrm{AU} / \mathrm{ml}$ after $24 \mathrm{~h}$ of methanol induction. However, the antimicrobial activity decreased from 48 to $72 \mathrm{~h}$. No antibacterial activity was detected in the supernatants of $P$. pastoris X-33 (pPIC$\mathrm{Z} \alpha \mathrm{A})$. The results of the MALDI-TOF MS for fermentation supernatants indicated that the molecular weight of rEntA was 4,830.1 Da, which was consistent with its theoretical value of 4,829 Da (Figure 2E).

To increase the production of rEntA, high-density fermentation of the recombinant yeast was performed using a 5-L fermenter. Although the total supernatant protein and biomass reached $365 \mathrm{mg} / \mathrm{l}$ and $343 \mathrm{~g} / \mathrm{l}$ after induction for $90 \mathrm{~h}$, the maximal antimicrobial activity was $51200 \mathrm{AU} / \mathrm{ml}(180 \mathrm{mg} / \mathrm{l})$ after induction for $24 \mathrm{~h}$ (Figure 2C), which was 8-fold higher than that found at the shake-flask level. Figures $2 \mathrm{~B}$ and D clearly showed that $\mathrm{rEntA}$ was rapidly degraded after $72 \mathrm{~h}$ of induction. Moreover, the expression of rEntA in the fermenter could be detected directly by Coomassie blue staining (Figure 2D), while its expression in the shake-flask could only be detected by silver staining (data not shown).

\section{Purification of rEntA}

The rEntA was purified from the ferment supernatant after a 24-h induction in a 5-L fermenter. The bacteriocin activity of $6.40 \times 10^{5} \mathrm{AU} / \mathrm{mg}$ with a 2.25 -fold increase was obtained after gel filtration. The purified rEntA was analyzed by Tricine-SDS-PAGE and showed a band at $4.8 \mathrm{kDa}$ representing the target protein band (Figure 2F), corresponding with its theoretical molecular weight.

\section{Antimicrobial spectrum of rEntA}

Only L. ivanovii ATCC19119, E. faecalis CGMCC1.130 and E. faecalis CGMCC1.2024 were sensitive to rEntA in the 16 tested strains. Other Gram-positive bacteria, such as E. faecium CGMCC1.2136, S. aureus ATCC25923, S. epidermidis ATCC26069, B. licheniformis CGMCC1.265, and B. coagulans CGMCC1.2407, were found to be resistant to rEntA. All of the Gram-negative bacteria strains were resistant to rEntA in this assay (Table 1). The MIC and MBC of rEntA against L. ivanovii ATCC19119 were $20 \mathrm{ng} / \mathrm{ml}$ and $80 \mathrm{ng} / \mathrm{ml}$, respectively, and were lower than those of ampicillin (390 $\mathrm{ng} / \mathrm{ml}$ and $1560 \mathrm{ng} / \mathrm{ml}$, respectively).

\section{In-vitro killing curve assay}

The time-killing kinetics curve showed that the amount of L. ivanovii ATCC19119 increased from 6.63 $\log _{10} \mathrm{CFU} / \mathrm{ml}$ to $9.48 \log _{10} \mathrm{CFU} / \mathrm{ml}$ within $10 \mathrm{~h}$ in the absence of rEntA. The decrease in the counts of $L$. ivanovii ATCC19119 varied considerably depending on the concentration of rEntA. For example, the maximum viability loss (MVL), which was approximately $0.44 \log _{10} \mathrm{CFU} / \mathrm{ml}$ ( $\sim 60 \%$ reduction in CFU), was reached within $2 \mathrm{~h}$ in $1 \times$ MIC of rEntA. The $2 \times$ MIC of rEntA could cause approximately $1.42 \log _{10} \mathrm{CFU} / \mathrm{ml}$ viability loss (96\% reduction) within $6 \mathrm{~h}$. Moreover, the MVL of L. ivanovii treated by rEntA at $4 \times$ MIC was approximately 2.03 $\log _{10} \mathrm{CFU} / \mathrm{ml}$ (>99\% reduction in CFU) within $4 \mathrm{~h}$. Although rEntA could inhibit the growth of L. ivanovii ATCC19119, the survivors resumed growth at $1 \times$ and $2 \times \mathrm{MIC}$ of rEntA and $2 \times$ MIC ampicillin for L. ivanovii ATCC19119 after MVL was achieved (Figure 3). However, L. ivanovii ATCC19119 treated by $4 \times$ MIC of rEntA did not show re-growth within $10 \mathrm{~h}$, revealing that $80 \mathrm{ng} / \mathrm{ml} \mathrm{rEntA}$ could effectively inhibit the growth of pathogenic bacteria for an extended time.

\section{Effects of $\mathrm{pH}$, temperature, proteolytic enzymes and $\mathrm{NaCl}$} on the activity of rEntA

As shown in Figure 4A, rEntA was highly stable at a wide range of $\mathrm{pH}$ values. The activity of rEntA was maintained completely within a $\mathrm{pH}$ range of $2-8$ at $37^{\circ} \mathrm{C}$ for $12 \mathrm{~h}$ and was $75 \%$ retained even after incubation at a $\mathrm{pH}$ of 10 for $12 \mathrm{~h}$. Furthermore, the antimicrobial activity of rEntA was not affected by heat treatment at 37, 60,80 and $100^{\circ} \mathrm{C}$ for $1 \mathrm{~h}$ under acid conditions $(\mathrm{pH} 2$ and 4 ) (Figure $4 \mathrm{~B}$ ). The residual activity decreased to $20 \%$ at a $\mathrm{pH}$ of 10 at $80^{\circ} \mathrm{C}$, to $50 \%$ at a $\mathrm{pH}$ of 6,8 at $100^{\circ} \mathrm{C}$, and to $10 \%$ at a $\mathrm{pH}$ of 10 at $100^{\circ} \mathrm{C}$. In addition, the antimicrobial activity of rEntA was completely abolished by pepsin and trypsin treatment, but it retained $16.7 \%$ of initial antimicrobial activity after papain treatment at $37^{\circ} \mathrm{C}$ for $1 \mathrm{~h}$ (Figure 4C).

The antimicrobial activity of rEntA against L. ivanovii ATCC19119 was slightly enhanced by the addition of 25 and $50 \mathrm{mM} \mathrm{NaCl}$ (Figure 5). The lowest amount of 2.43 $\log _{10} \mathrm{CFU} / \mathrm{ml}$ was observed with a treatment of rEntA $(12,800 \mathrm{AU} / \mathrm{ml})$ in $25 \mathrm{mM} \mathrm{NaCl}(44.52 \%$ of that at 


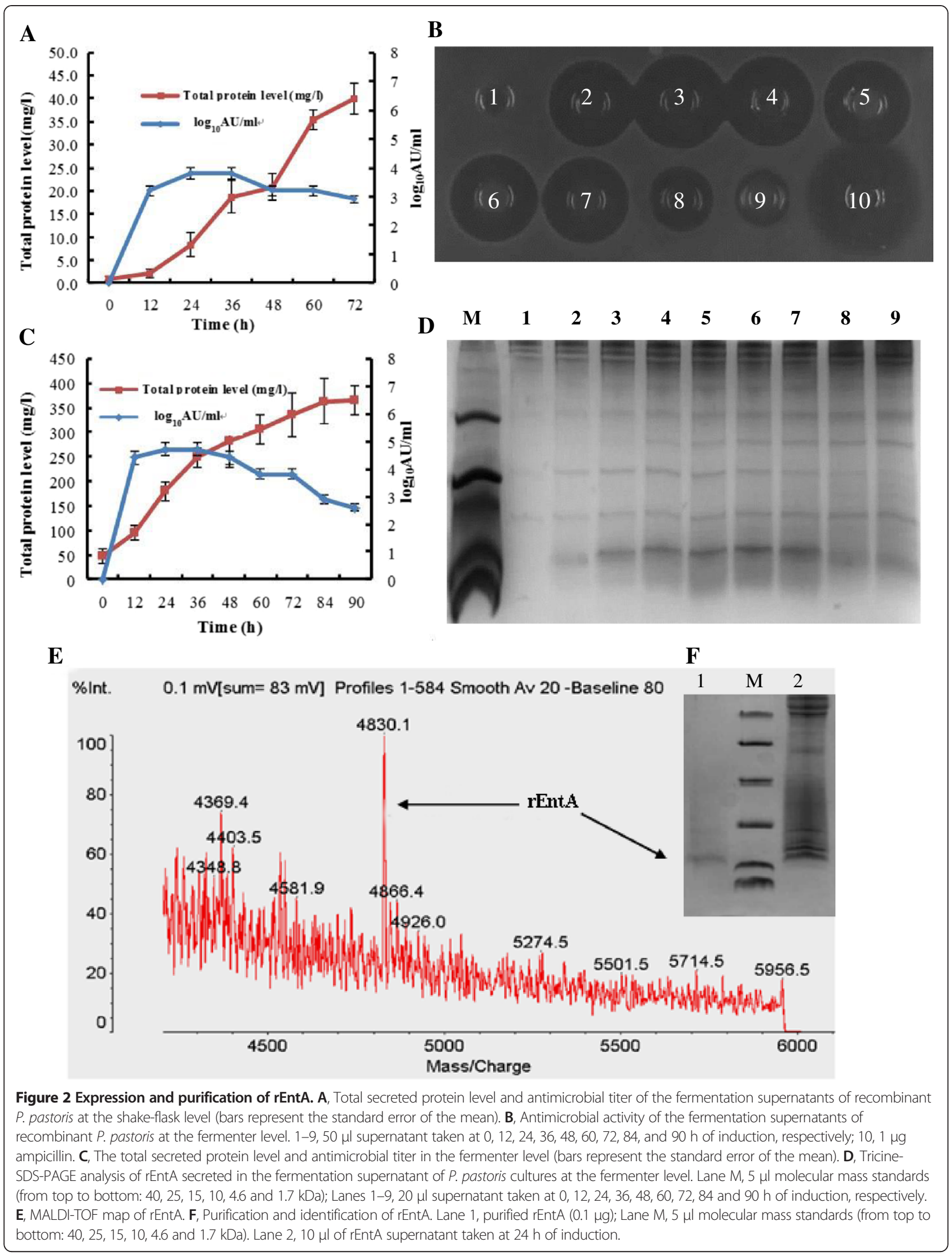


Table 1 Antimicrobial spectrum of rEntA

\begin{tabular}{ll}
\hline Strains & Antimicrobial activity \\
\hline Gram-positive & + \\
Listeria ivanovii ATCC19119 & - \\
Enterococcus faecium CGMCC1.2136 & + \\
Enterococcus faecalis CGMCC1.130 & + \\
Enterococcus faecalis CGMCC1.2024 & - \\
Staphylococcus aureus ATCC 25923 & - \\
Staphylococcus epidermidis ATCC26069 & - \\
Bacillus licheniformis CGMCC1.265 & - \\
Bacillus coagulans CGMCC1.2407 & - \\
Bacillus subtilis ATCC6633 & - \\
Lactococcus lactis (Stored in our lab) & - \\
Bifidobacterium bifidum CGMCC1.2212 & - \\
Gram-negative & - \\
E. coli ER2566 & - \\
E. coli CVCC 195 & - \\
E. coli CMCC 44102 & - \\
Seudomonas aeruginosa CVCC 2087 & - \\
\hline
\end{tabular}

Note: " + " refers to positive antimicrobial activity (inhibition zone $>6 \mathrm{~mm}$ ) "-" refers to negative antimicrobial activity (inhibition zone $\leq 6 \mathrm{~mm}$ ).

$0 \mathrm{mM} \mathrm{NaCl}$ ). The other treatments, from $100-$ $400 \mathrm{mM} \mathrm{NaCl}$, had no significant effect on the bactericidal ability of rEntA (Figure 5). In the controls without rEntA, growth was not influenced by $\mathrm{NaCl}(0-$ $400 \mathrm{mM}$ ) (Figure 5).

\section{Discussion}

Bacteriocin has attracted attention in recent years for its potential application as a food preservative and therapeutic antimicrobial agent [20]. However, low production of these bacteriocins by native strains cannot meet the requirements of commercial applications. Moreover, some Enterococci strains were recognized as opportunistic pathogens associated with lots of infections [21]. Attempts to produce bacteriocins by using safe heterologous hosts have been undertaken in recent years [17,22,23], including some typical expression systems such as $E$. coli, $L$. lactis, and P. pastoris. Although E. coli and L. lactis are widely used in heterologous protein expression because of their easy operation and safety $[14,24]$, they are not suitable for bacteriocins due to toxicity to the host [25] and low recovery percentages from the fusion protein [26]. Many bacteriocins, such as enterocin P [17], hiracin JM79 [27], enterocin L50 [28], pediocin PA-1 [29] and EntA [18], have been expressed as active forms in $P$. pastoris, but their expression levels remained low (below $280 \mathrm{mg} / \mathrm{l}$ ). It is known that codon optimization is a useful strategy to increase the yield of target protein during heterogeneous expression. Many antimicrobial peptides, such as plectasin [30], NZ2114 [31] and AgPlectasin [32], were expressed with high production through codon-usage optimization in our laboratory. In addition, Divercin V41, a class IIa bacteriocins was also expressed through this system [33]. These cases encouraged us to use codon optimization to break through the bottleneck of low yield in heterologous expression of EntA. The total protein level in the

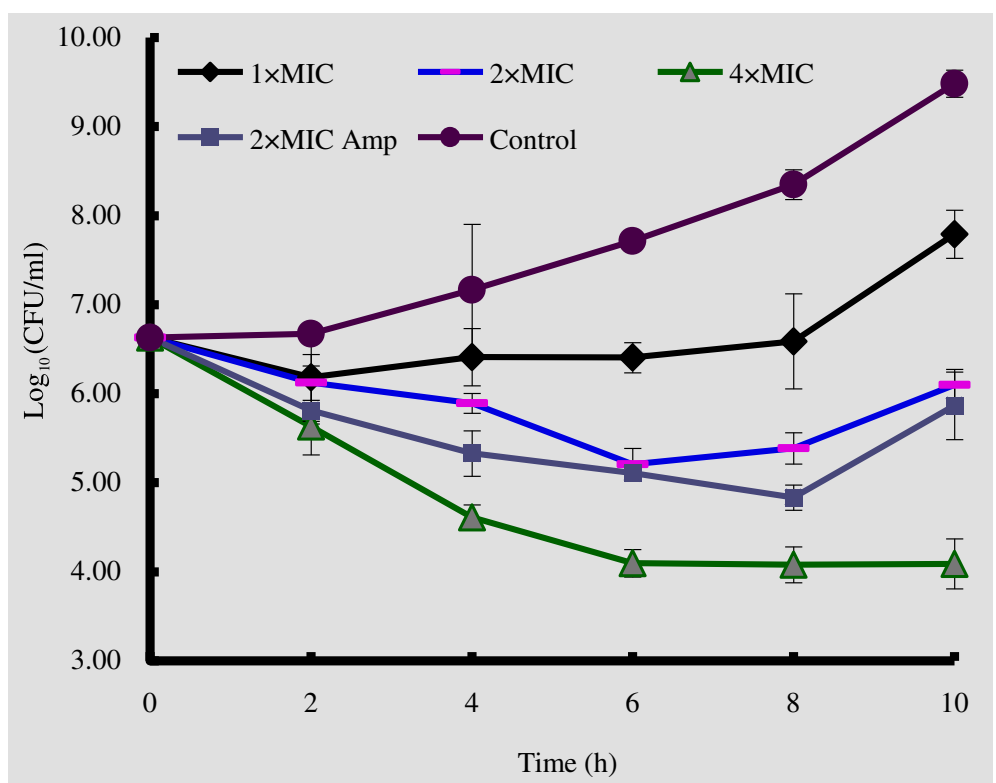

Figure 3 Time-kill curves of rEntA. L. ivanovii ATCC19119 was incubated in the presence of medium alone or in the presence of 1x, 2x, or 4x MIC of rEntA. Ampicillin of $2 \times$ MIC was used as a positive control. Three duplicate observations were made; bars represent the standard error of the mean. 


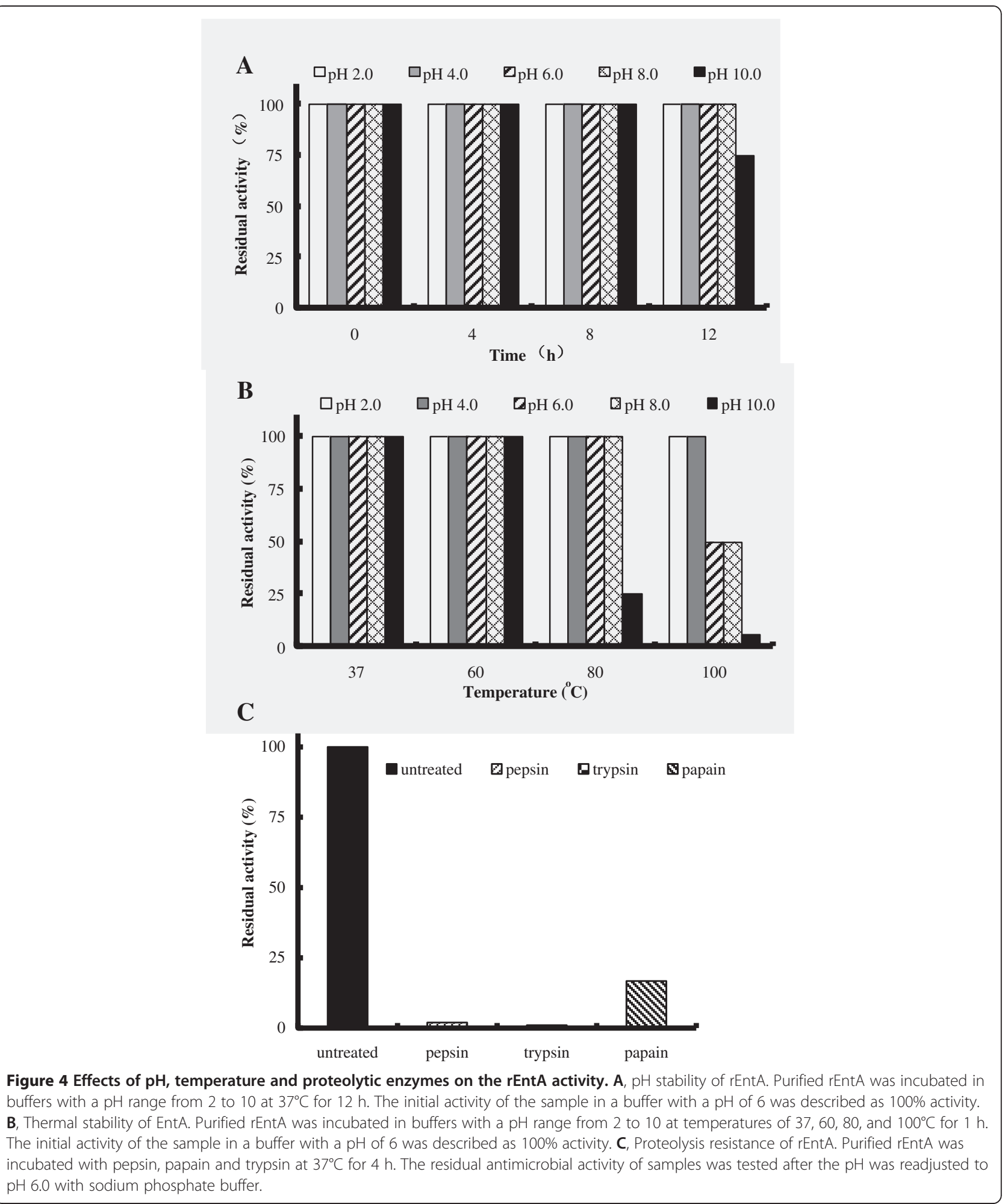

supernatant reached $180 \mathrm{mg} / \mathrm{l}$ with the activity of $51,200 \mathrm{AU} / \mathrm{ml}$ at $24 \mathrm{~h}$ of induction in 5-L fermenter level (Figure 2C) after the gene was optimized. Although the yield of target peptide was still low, and even lower than $280 \mathrm{mg} / \mathrm{l}$ as the highest result of expression in case of enterocin L50 in P. pastoris [28], it was much higher than that of Pediocin PA-1 $(0.4 \mathrm{mg} / \mathrm{l})$, Enterocin P $(0.006 \mathrm{mg} / \mathrm{l})$, Divercin V41 (23 mg/l) and EntA (0.027 mg/l) expressed in $E$. coli and L. lactis $[14,22,33]$. Furthermore, the production of rEntA increased 2.99-times compared with its 


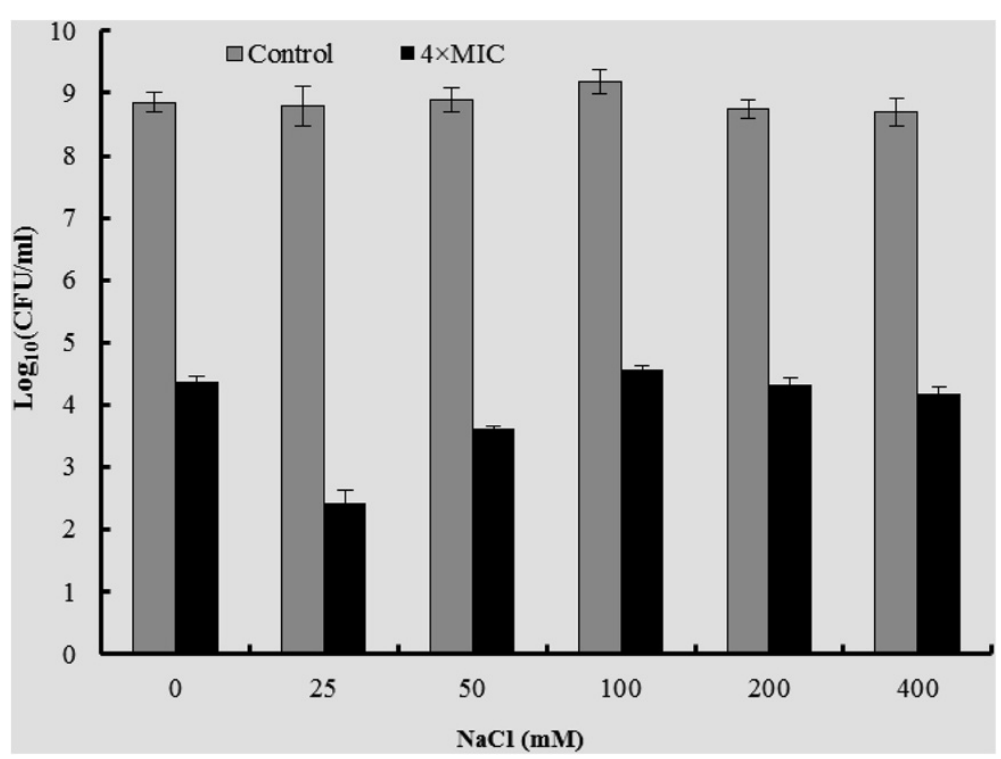

Figure 5 Effect of $\mathrm{NaCl}$ concentration on the activity of rEntA. Control: L. ivanovii ATCC19119 was incubated in the absence of rEntA. $4 \times$ MIC: L. ivanovii ATCC19119 was incubated in the presence of rEntA at $4 \times$ MIC.

native sequence expressed in $P$. pastoris (45.1 mg/l), which indicated codon optimization is a good tool to enhance expression efficiency and level in P. pastoris, and at the same time, it also left a large room to improve in future work at the similar aim and technical scheme.

However, the maximal activity of rEntA in the supernatant was reached at an early stage (24 h) of induction (Figure 2C). This is similar to previous results in which the highest level of rEntA was reached at $36 \mathrm{~h}$. An even earlier peak of rEntA at $6 \mathrm{~h}$ was observed in other yeasts such as Kluyveromyces lactis and Hansenula polymorpha [18]. Obviously, its final successful application suffered from this strong decomposition in the supernatant at an earlier period of expression related to the possible disruption of rEntA to host cells and the proteolysis of the target protein. The latter situation was reported in "collagen-like" bacteriocin with a high cleavage by collagenase [29]. However, the exact mechanism of the above described early degradation and its solution should be further studied.

A series of methods, such as ion exchange chromatography (SP and CM FF), hydrophobic exchange chromatography (Phenyl HP), and gel filtration (Superose 12), were attempted to purify rEntA in this study. Only gel filtration could purify rEntA with a yield of $3.02 \mathrm{mg} / \mathrm{l}$ (Figure 2F) after attempts with SP FF, CM FF, and phenyl HP in which almost all rEntA was lost in the penetration peak (data not shown) due to unknown reasons.

Although there are different antibacterial spectrums between class IIa bacteriocins, they consistently have particularly high antibacterial activity against Listeria - the most common pathogen in food - at nanomolar concentrations [1]. The MICs of purified native EntA from $E$. faecium T136 against Listerias ranged from 40 to $120 \mathrm{ng} / \mathrm{ml}$ [34]. Similarly, rEntA also showed a narrow antibacterial spectrum (Table 1 ) including $L$. ivanovii ATCC19119, and with a low MIC value of $20 \mathrm{ng} / \mathrm{ml}$, it is approximately 20 -fold lower than that of ampicillin (390 $\mathrm{ng} / \mathrm{ml})$. The re-growth after MVL achievement was a common phenomenon when the Listeria was treated with bacteriocins such as EntA, pediocin, sakacin $A$ and enterococcin EFS2 in relatively low concentrations $(1 \times$ or $2 \times \mathrm{MIC})$ [3], but we found no re-growth after MVL within $10 \mathrm{~h}$ when $4 \times$ MIC $\mathrm{rEnA}$ was used with the Listeria (Figure 3), indicating that higher concentrations of rEnA are essential to inhibit the multiplication of Listeria.

The bactericidal activity and overall structure of Pediocin PA-1 and piscicolin 126 were well maintained at higher temperatures $[35,36]$. The native EntA was stable at $100^{\circ} \mathrm{C}$ and acidic $\mathrm{pH}$ conditions [37]. We found that rEntA also exhibited high stability under a wide range of temperatures $\left(37-80^{\circ} \mathrm{C}\right)$ and $\mathrm{pH}$ levels (2-8) (Figure 4). These properties were potentially due to the higher cysteine content of the antimicrobial peptides [38], similar to the EntA containing four cysteine residues. In addition, the antimicrobial activity of some bacteriocins (nisin, sakacin $\mathrm{P}$ and curvacin A) was significantly enhanced with the addition of $\mathrm{NaCl}$ from 0 to $1.17 \mathrm{M}$ [39]. However, the activity of rEntA against Listeria was enhanced only at low $\mathrm{NaCl}$ concentrations (25 and $50 \mathrm{mM})$. Despite the unknown mechanisms of the above differential effects, the high stability of rEntA over wide 
ranges of temperature, $\mathrm{pH}$, and $\mathrm{NaCl}$ concentration supports its use as a food preservative and drug candidate.

Due to the high content of basic and aromatic amino acids in class IIa bacteriocins, pediocin PA-1, enterocin $B$, plantaricin 423 and native EntA were very sensitive to the digestive proteases trypsin and pepsin [11,40,41]. Similarly, the purified rEntA, with $12.76 \%$ basic amino acids and $10.63 \%$ aromatic amino acids, was inactivated with trypsin and pepsin (Figure 4C). This high sensitivity to digestive proteases of rEntA contributes to its safety in foods and drugs, during and after oral administration.

\section{Conclusion}

In conclusion, rEntA, as an antimicrobial agent with merit, could selectively kill important and pathogenic Listeria and retain bio-activity over a wide range of $\mathrm{pH}$ values, temperature and $\mathrm{NaCl}$ concentrations. These excellent antibacterial properties make it a potential candidate as a food preservative and therapeutic antimicrobial agent. rEntA was successfully expressed in P. pastoris X33 at the highest level of 51,200 AU/ml and was purified through a gel filtration column. This yeast system may be a feasible technological approach to produce rEntA as a potent anti-Listeria agent after further optimization.

\section{Methods}

\section{Strains and vectors}

Escherichia coli DH5 $\alpha$, Pichia pastoris X-33 and pPIC$\mathrm{Z} \alpha \mathrm{A}$ were purchased from Invitrogen (Beijing, China). Target strains for the antimicrobial activity assays are listed in Table 2. Restriction enzymes were purchased from New England Biolabs (NEB, Beijing, China). The kits for plasmid extraction and DNA purification were purchased from Tiangen (Beijing, China). Other chemical reagents used in this research were all of analytical grade.

Construction of the expression vector and transformation The optimized EntA gene (GenBank accession No. KJ155693) was generated by the 'ReverseTranslateTool' (http://www.bioinformatics.org/sms2/rev_trans.html) according to the codon usage of $P$. pastoris (http://www. kazusa.or.jp/codon/). To express the target protein with a native $\mathrm{N}$-terminus, the Kex2 signal cleavage site was fused to the EntA sequence. The designed sequence was synthesized by Sangon Biotech (Shanghai, China) and digested using XhoI and XbaI. Resulting DNA fragments were ligated into pPICZ $\alpha$ A to generate the recombinant vector $\mathrm{pPICZ} \alpha \mathrm{A}$-EntA. The latter was transformed into E. coli $\mathrm{DH} 5 \alpha$, and positive transformants were confirmed by DNA sequencing. The recombinant plasmid was linearized with PmeI and transformed into P. pastoris X-33 competent cells by electroporation [30]. Positive transformants were screened on YPDS medium containing
Table 2 Strains used in the antimicrobial activity assays

\begin{tabular}{|c|c|}
\hline Strains & Source \\
\hline \multicolumn{2}{|l|}{ Gram-positive } \\
\hline Listeria ivanovii ATCC19119 & $\mathrm{ClCC}^{\mathrm{a}}$ \\
\hline Enterococcus faecium CGMCC1.2136 & $\mathrm{CGMCC}^{\mathrm{b}}$ \\
\hline Enterococcus faecalis CGMCC1.130 & CGMCC \\
\hline Enterococcus faecalis CGMCC1.2024 & CGMCC \\
\hline Staphylococcus aureus ATCC 25923 & $\mathrm{CVCC}^{\mathrm{C}}$ \\
\hline Staphylococcus epidermidis ATCC26069 & CVCC \\
\hline Bacillus licheniformis CGMCC1.265 & CGMCC \\
\hline Bacillus coagulans CGMCC1.2407 & CGMCC \\
\hline Bacillus subtilis ATCC6633 & CVCC \\
\hline Lactococcus lactis & Stored in our lab \\
\hline Bifidobacterium bifidum CGMCC1.2212 & CGMCC \\
\hline \multicolumn{2}{|l|}{ Gram-negative } \\
\hline Escherichia. coli ER2566 & CGMCC \\
\hline Escherichia. coli CVCC 195 & CVCC \\
\hline Escherichia. coli CMCC 44102 & $\mathrm{CMCC}^{\mathrm{d}}$ \\
\hline Pseudomonas aeruginosa CVCC 2087 & CVCC \\
\hline Salmonella enteritidis CVCC3377 & CVCC \\
\hline
\end{tabular}

$100 \mu \mathrm{g} / \mathrm{ml}$ of zeocin and further confirmed by colonyPCR.

\section{Expression of rEntA at the shake-flask level}

The positive transformants were grown in BMGY medium until the cultures reached an OD600 $\mathrm{nm}$ of $5.0-6.0$ at $30^{\circ} \mathrm{C}$. Cells were harvested by centrifugation at $4000 \mathrm{rpm}$ for $10 \mathrm{~min}$ and resuspended in BMMY medium to an OD600 nm of 1.0. Methanol was added daily to a final concentration of approximately $0.5 \%$. Samples were taken at 0, 12, 24, 36, 48, 60 and $72 \mathrm{~h}$ for analysis.

\section{Expression of rEntA at the fermenter level}

A single colony of $P$. pastoris X-33 (pPICZ $\alpha \mathrm{A}-$ EntA) was grown in $10 \mathrm{ml}$ of YPD medium at $30^{\circ} \mathrm{C}$ overnight. The culture was inoculated into $200 \mathrm{ml}$ fresh YPD medium and cultivated at $29^{\circ} \mathrm{C}$ to an OD600 nm of approximately 6.0. The 200-ml seed culture was transferred into a 5-L fermenter (Sartorius StedimBiotech) containing $1.8 \mathrm{~L}$ of basal salt medium with $45 \mathrm{~g} / \mathrm{L}$ of $\mathrm{NH}_{4} \mathrm{H}_{2} \mathrm{PO}_{4}$, $20 \mathrm{~g} / \mathrm{L} \mathrm{K}_{2} \mathrm{SO}_{4}, 0.4 \mathrm{~g} / \mathrm{L} \mathrm{CaSO}_{4}, 15 \mathrm{~g} / \mathrm{L} \mathrm{MgSO}_{4} 7 \mathrm{H}_{2} \mathrm{O}, 6$ $\mathrm{g} / \mathrm{L} \mathrm{KH}_{2} \mathrm{PO}_{4}, 1.5 \mathrm{~g} / \mathrm{L} \mathrm{KOH}$, and $200 \mathrm{ml} \mathrm{45 \%} \mathrm{w/v} \mathrm{glucose.}$ The initial fermentation was a glucose batch phase (approximately $18 \mathrm{~h}$ ). After exhaustion of the glucose, $50 \% \mathrm{w} / \mathrm{v}$ glucose was added for $6 \mathrm{~h}$ at a feed rate of $36 \mathrm{ml} / \mathrm{h}$. After the glucose was exhausted, methanol was 
supplied from 2 to $12 \mathrm{ml} / \mathrm{h}$. The whole fermentation period was performed at $29^{\circ} \mathrm{C}$. During the glucose batch and glucose-fed phases, the $\mathrm{pH}$ was kept at 5.0 and increased to 5.5 at the methanol induction phase [42]. The protein in the supernatant was determined by the Bradford protein assay (Tiangen, Beijing, China) and TricineSDS-PAGE [43].

\section{Purification of rEntA}

The supernatant with rEntA from $P$. pastoris $\mathrm{X}-33$ (pPICZ $\alpha A-E n t A) ~ X-33$ was desalted by a gel filtration column (Sephadex G-25) with a flow rate of $2 \mathrm{ml} / \mathrm{min}$ and then freeze-dried and dissolved in $100 \mathrm{mM}$ of ammonium acetate buffer. The sample was passed through a gel filtration column (Superose 12) and eluted with the same buffer at a flow rate of $0.5 \mathrm{ml} / \mathrm{min}$. Purified rEntA was further lyophilized to remove ammonium acetate.

\section{Antimicrobial activity assay}

Tested strains including L. ivanovii, E. faecalis, and E. faecium were grown in Mueller-Hinton (MH) broth containing 3\% fetal bovine serum (FBS). S. epidermidis, B. subtilis, L. lactis, B. bifidum, B. licheniformis, B. coagulans and S. aureus were grown in $\mathrm{MH}$ broth. $P$. aeruginosa, E. coli and $S$. enteritidis were grown in LB medium. All tested strains were grown to 0.4 of OD600 nm at $37^{\circ} \mathrm{C}$. One hundred microliters of the cell suspension was inoculated into $50 \mathrm{ml}$ of preheated medium containing 1.5\% agar. This was rapidly mixed and poured into a Petri dish. Sterile Oxford cups were put on the surface of the solidified media. Each cup was filled with $50 \mu \mathrm{l}$ of samples [30].

Titer assays were used to quantify the antimicrobial activity of rEntA according to the method of Liu [12]. The titer was expressed as arbitrary units (AU/ml). One arbitrary unit (AU) was defined as the reciprocal of the highest dilution showing a clear zone of inhibition to the indicator strain. When a clear inhibition zone was followed by a turbid one, the critical dilution was taken to be the average of the final two dilutions.

Minimal inhibitory concentrations (MICs) and Minimum bactericidal concentrations (MBCs) assays were determined using the microtiter broth dilution method [30]. Ampicillin was also tested with the same concentration gradient as a positive control. All tests were performed in triplicate.

\section{In-vitro killing curve assay}

To evaluate the antibacterial activity of rEntA against $L$. ivanovii ATCC19119, a time-kill assay was performed as described by the methods of Mao [32]. In addition, tubes with only bacterial inoculum were used as growth controls. All experiments were performed in triplicate.

\section{Effects of $\mathrm{pH}$, temperature, proteases and $\mathrm{NaCl}$ on the activity of rEntA}

The effects of $\mathrm{pH}$, temperature and proteases on rEntA activity were determined as described previously $[30,44]$ with the following modifications: 1) The titer of purified rEntA used in the test was $12,800 \mathrm{AU} / \mathrm{ml}$; 2) The initial activity of sample in the buffer with a $\mathrm{pH}$ of 6 was taken as $100 \%$ activity for $\mathrm{pH}$ and thermal stability assays; and 3) The residual antimicrobial activity of samples was tested after the $\mathrm{pH}$ was readjusted to 6.0 with sodium phosphate buffer ( $\mathrm{pH}$ 6.0) for the proteolytic sensibility assay.

To evaluate the effect of $\mathrm{NaCl}$ concentration on the activity of rEntA, an overnight culture of $L$. ivanovii ATCC19119 was diluted to $10^{5-6} \mathrm{CFU} / \mathrm{ml}$ in fresh MHB medium (3\% FBS). Ten microliters of purified rEntA and $10 \mu \mathrm{l}$ of $\mathrm{NaCl}$ solution were added to $80 \mu \mathrm{l}$ of diluted cell culture. The final rEntA concentration was $4 \times \mathrm{MIC}$, and the final $\mathrm{NaCl}$ concentrations were $0,25,50,100$, 200, and $400 \mathrm{mM}$. Samples without rEntA were used as controls. All samples were incubated at $37^{\circ} \mathrm{C}$ for $10 \mathrm{~h}$. The CFU of tested strains was determined. All tests were performed in triplicate.

\section{Competing interests}

The authors declare that they have no competing interests.

\section{Authors' contributions}

$\mathrm{XH}$ carried out the reference collection and analysis, most experimental running of whole expermental work; RM participated partial experimental design, method improvement and partial paper writing; $Y Z$ was charge of expression and mainly fermentor running; DT was charge of codon optimization and all materials preparation; XW was charge of partial DNA cloning and PCR techinque, and partial result analysis; DX participated partial peptide purification; $\mathrm{JH}$ corrected partial techincal design on microbiological methods; JW participated and coordinated all sections of this work, design and running, results analysis and disscussion, paper writing and correction. All authors read and approved the final manuscript.

\section{Acknowledgments}

The authors wish to acknowledge Prof. Yang Fuquan, Ph.D., in the Proteomics Platform Laboratory, Institute of Biophysics, Chinese Academy of Sciences, for his coordination of the MALDI-TOF MS analysis. In addition, all other experiments described in this paper were run in the Gene Engineering Laboratory, Feed Research Institute, Chinese Academy of Agricultural Sciences. This work was supported by the National Natural Science Foundation of China (No. 31372346, No. 31302004 and No. 30972125), the Project of National Support Program for Science and Technology in China (No. 2013BAD10B02 and No. 2011BAD26B02), and the AMP Direction of Innovation Program of Agric Sci \& Tech in CAAS (2013-2017).

\section{Author details}

${ }^{1}$ Key Laboratory of Feed Biotechnology, Ministry of Agriculture, Beijing 100081, China. ${ }^{2}$ Gene Engineering Laboratory, Feed Research Institute, Chinese Academy of Agricultural Sciences, 12 Zhongguancun Nandajie St., Haidian District, Beijing 100081, P. R. China. ${ }^{3}$ College of Life Science, Engineering Research Center of Industrial Microbiology, Fujian Normal University, Fuzhou 350108, China

Received: 4 March 2014 Accepted: 12 August 2014

Published: 28 August 2014

\section{References}

1. Lohans CT, Vederas JC: Development of Class Ila bacteriocins as therapeutic agents. Int J Microbiol 2012, 2012:1-13. 
2. Cotter PD, Hill C, Ross RP: Bacteriocins: developing innate immunity for food. Nat Rev Microbiol 2005, 3:777-788.

3. Ennahar S, Deschamps N: Anti-Listeria effect of enterocin A, produced by cheese-isolated Enterococcus faecium EFM01, relative to other bacteriocins from lactic acid bacteria. J Appl Microbio/ 2000, 88:449-457.

4. Cotter PD, Ross RP, Hill C: Bacteriocins-a viable alternative to antibiotics? Nat Rev Microbiol 2012, 11:95-105.

5. Blay GL, Lacroix C, Zihler A, Fliss I: In vitro inhibition activity of nisin A, nisin Z, pediocin PA-1 and antibiotics against common intestinal bacterial. Lett Appl Microbiol 2007, 45:252-257.

6. Engelbrecht F, Domínguez-Bernal G, Hess J, Dickneite C, Greiffenberg L, Lampidis R, Raffelsbauer D, Daniels JJ, Kreft J, Kaufmann SH: A nove PrfA-regulated chromosomal locus, which is specific for Listeria ivanovii, encodes two small, secreted internalins and contributes to virulence in mice. Mol Microbiol 1998, 30:405-417.

7. Bodro M, Paterson DL: Listeriosis in patients receiving biologic therapies. Eur J Clin Microbiol Infect Dis 2013, 32:1225-1230.

8. Scallan E, Hoekstra RM, Angulo FJ, Tauxe RV, Widdowson MA, Roy SL, Jones $J$, Griffin PM: Foodborne illness acquired in the United States-major pathogensl. Emerg Infect Dis 2011, 17:7-15.

9. Fallah AA, Saei-Dehkordi SS, Mahzounieh M: Occurrence and antibiotic resistance profiles of Listeria monocytogenes isolated from seafood products and market and processing environments in Iran. Food Control 2013, 34:630-636.

10. Aymerich T, Holo H, Håvarstein LS, Hugas M, Garriga M, Nes IF: Biochemical and genetic characterization of enterocin A from Enterococcus faecium, a new antilisterial bacteriocin in the pediocin family of bacteriocins. Appl Environ Microbiol 1996, 62:1676-1682.

11. Herranz C, Casaus P, Mukhopadhyay S, Martınez J, Rodrıguez J, Nes I, Hernández P, Cintas L: Enterococcus faecium P21: a strain occurring naturally in dry-fermented sausages producing the class II bacteriocins enterocin A and enterocin B. Food Microbiol 2001, 18:115-131.

12. Liu L, O'Conner P, Cotter P, Hill C, Ross R: Controlling Listeria monocytogenes in cottage cheese through heterologous production of enterocin A by Lactococcus lactis. J Appl Microbiol 2008, 104:1059-1066.

13. Rehaiem A, Martínez B, Manai M, Rodríguez A: Technological performance of the enterocin A producer Enterococcus faecium MMRA as a protective adjunct culture to enhance hygienic and sensory attributes of traditional fermented milk 'Rayeb'. Food Bioprocess Tech 2012, 5:2140-2150.

14. Gutiérrez J, Criado R, Citti R, Martín M, Herranz C, Nes IF, Cintas LM, Hernández PE: Cloning, production and functional expression of enterocin $\mathrm{P}$, a sec-dependent bacteriocin produced by Enterococcus faecium P13, in Escherichia coli. Int J Food Microbiol 2005, 103:239-250.

15. Ingham A, Sproat K, Tizard M, Moore R: A versatile system for the expression of nonmodified bacteriocins in Escherichia coli. J Appl Microbiol 2005, 98:676-683.

16. Le Loir Y, Azevedo V, Oliveira SC, Freitas DA, Miyoshi A, Bermúdez-Humarán $L G$, Nouaille S, Ribeiro LA, Leclerca S, Gabriel JE: Protein secretion in Lactococcus lactis: an efficient way to increase the overall heterologous protein production. Microb Cell Fact 2005, 4:2.

17. Gutiérrez J, Criado R, Martín M, Herranz C, Cintas LM, Hernández PE: Production of enterocin $\mathrm{P}$, an antilisterial pediocin-like bacteriocin from Enterococcus faecium P13, in Pichia pastoris. Antimicrob Agents Chemother 2005, 49:3004-3008.

18. Borrero J, Kunze G, Jiménez JJ, Böer E, Gútiez L, Herranz C, Cintas LM, Hernández PE: Cloning, production, and functional expression of the bacteriocin enterocin A, produced by Enterococcus faecium T136, by the Yeasts Pichia pastoris, Kluyveromyces lactis, Hansenula polymorpha, and Arxula adeninivorans. Appl Environ Microbiol 2012, 78:5956-5961.

19. Li Y, Zhang B, Chen X, Cao Y: Improvement of Aspergillus sulphureus endo- $\beta-1,4$-xylanase expression in Pichia pastoris by codon optimization and analysis of the enzymic characterizationl. Appl Biochem Biotech 2010 160:1321-1331.

20. Hassan M, Kjos M, Nes I, Diep D, Lotfipour F: Natural antimicrobial peptides from bacteria: characteristics and potential applications to fight against antibiotic resistance. J Appl Microbiol 2012, 113:723-736.

21. Franz CM, Van Belkum MJ, Holzapfel WH, Abriouel H, Galvez A: Diversity of enterococcal bacteriocins and their grouping in a new classification scheme. FEMS Microbiol Rev 2007, 31:293-310.

22. Martínez JM, Kok J, Sanders JW, Hernández PE: Heterologous coproduction of enterocin A and pediocin PA-1 by Lactococcus lactis: detection by specific peptide-directed antibodies. Appl Environ Microbiol 2000, 66:3543-3549.

23. Klocke M, Mundt $K$, Idler F, Jung S, Backhausen JE: Heterologous expression of enterocin A, a bacteriocin from Enterococcus faecium, fused to a cellulose-binding domain in Escherichia coli results in a functional protein with inhibitory activity against Listeria. App/ Microbiol Biotechnol 2005, 67:532-538.

24. Borrero J, Jiménez JJ, Gútiez L, Herranz C, Cintas LM, Hernández PE: Protein expression vector and secretion signal peptide optimization to drive the production, secretion, and functional expression of the bacteriocin enterocin A in lactic acid bacteria. J Biotechnol 2011, 156:76-86.

25. Zorko M, Japelj B, Hafner-Bratkovic I, Jerala R: Expression, purification and structural studies of a short antimicrobial peptide. BBA-Biomembranes 2009, 1788:314-323.

26. Kim J, Jang S, Yu B, Sung B, Cho J, Kim S: High-level expression of an antimicrobial peptide histonin as a natural form by multimerization and furin-mediated cleavage. Appl Microbiol Biotechnol 2008, 78:123-130.

27. Sánchez J, Borrero J, Gómez-Sala B, Basanta A, Herranz C, Cintas L, Hernández PE: Cloning and heterologous production of hiracin JM79, a Sec-dependent bacteriocin produced by Enterococcus hirae DCH5, in lactic acid bacteria and Pichia pastoris. Appl Environ Microbiol 2008, 74:2471-2479.

28. Basanta A, Gómez-Sala B, Sánchez J, Diep DB, Herranz C, Hernández PE, Cintas LM: Use of the yeast Pichia pastoris as an expression host for secretion of enterocin L50, a leaderless two-peptide (L50A and L50B) bacteriocin from Enterococcus faecium L50. Appl Environ Microbio 2010, 176:3314-3324.

29. Beaulieu L, Groleau D, Miguez CB, Jetté J-F, Aomari H, Subirade M: Production of pediocin PA-1 in the methylotrophic yeast Pichia pastoris reveals unexpected inhibition of its biological activity due to the presence of collagen-like material. Protein Expr Purif 2005, 43:111-125.

30. Zhang J, Yang Y, Teng D, Tian Z, Wang S, Wang J: Expression of plectasin in Pichia pastoris and its characterization as a new antimicrobial peptide against Staphyloccocus and Streptococcus. Protein Expr Purif 2011 78:189-196

31. Zhang Y, Teng D, Mao R, Wang X, Xi D, Hu X, Wang J: High expression of a plectasin-derived peptide NZ2114 in Pichia pastoris and its pharmacodynamics, postantibiotic and synergy against Staphylococcus aureus. Appl Microbiol Biotechnol 2014, 98:681-694.

32. Mao R, Teng D, Wang X, Xi D, Zhang Y, Hu X, Yang Y, Wang J: Design, expression, and characterization of a novel targeted plectasin against methicillin-resistant Staphylococcus aureus. App/ Microbiol Biotechnol 2013, 97:3991-4002

33. Richard C, Drider D, Elmorjani K, Marion D, Prévost H: Heterologous expression and purification of active Divercin V41, a Class Ila bacteriocin encoded by a synthetic gene in Escherichia coli. J Bacteriol 2004, 186:4276-4284.

34. Casaus P, Nilsen T, Cintas LM, Nes IF, Hernández PE, Holo H: Enterocin B, a new bacteriocin from Enterococcus faecium T136 which can act synergistically with enterocin A. Microbiology 1997, 143:2287-2294.

35. Kaur K, Andrew LC, Wishart DS, Vederas JC: Dynamic relationships among type lla bacteriocins: temperature effects on antimicrobial activity and on structure of the C-terminal amphipathic a helix as a receptor-binding region. Biochemistry 2004, 43:9009-9020.

36. Jack RW, Wan J, Gordon J, Harmark K, Davidson BE, Hillier AJ, Wettenhall R, Hickey MW, Coventry MJ: Characterization of the chemical and antimicrobial properties of piscicolin 126, a bacteriocin produced by Carnobacterium piscicola JG126. Appl Environ Microbiol 1996, 62:2897-2903.

37. Rehaiem A, Guerra NP, Belgacem ZB, Bernárdez PF, Castro LP, Manai M: Enhancement of enterocin A production by Enterococcus faecium MMRA and determination of its stability to temperature and $\mathrm{pH}$. Biochem Eng $J$ 2011, 56:94-106.

38. Yamada O, Sakamoto K, Tominaga M, Nakayama T, Koseki T, Fujita A, Akita $\mathrm{O}$ : Cloning and heterologous expression of the antibiotic peptide (ABP) genes from Rhizopus oligosporus NBRC 8631. Biosci Biotechnol Biochem 2005, 69:477-482.

39. Gänzle MG, Weber S, Hammes WP: Effect of ecological factors on the inhibitory spectrum and activity of bacteriocins. Int J Food Microbiol 1999, 46:207-217.

40. Reenen $\mathrm{V}$ : Isolation, purification and partial characterization of plantaricin 423, a bacteriocin produced by Lactobacillus plantarum. J Appl Microbiol 1998, 84:1131-1137. 
41. Rodriguez JM, Martinez Ml, Kok J: Pediocin PA-1, a wide-spectrum bacteriocin from lactic acid bacteria. Crit Rev Food Sci Nutr 2002, 42:91-121.

42. Bai X, Teng D, Tian Z, Zhu Y, Yang Y, Wang J: Contribution of bovine lactoferrin inter-lobe region to iron binding stability and antimicrobial activity against Staphylococcus aureus. BioMetals 2010, 23:431-439.

43. Schägger H: Tricine-SDS-PAGE. Nat Protoc 2006, 1:16-22.

44. Iwatani S, Zendo T, Yoneyama F, Nakayama J, Sonomoto K:

Characterization and structure analysis of a novel bacteriocin, lacticin Z produced by Lactococcus lactis QU 14. Biosci Biotechnol Biochem 2007, 71:1984-1992.

doi:10.1186/s12866-014-0220-8

Cite this article as: Hu et al:: Biotechnical paving of recombinant enterocin A as the candidate of anti-Listeria agent. BMC Microbiology 2014 14:220

\section{Submit your next manuscript to BioMed Central and take full advantage of:}

- Convenient online submission

- Thorough peer review

- No space constraints or color figure charges

- Immediate publication on acceptance

- Inclusion in PubMed, CAS, Scopus and Google Scholar

- Research which is freely available for redistribution 